\title{
Modelo de análise do perfil do consumidor de produtos orgânicos para definição da estratégia de marketing
}

O objetivo deste estudo é propor um modelo de análise que identifique o perfil do consumidor de alimentos orgânicos e, com o resultado encontrado, traçar a estratégia de marketing direcionada para este público consumidor, uma vez que na perspectiva atual do consumidor em optar por alimentos saudáveis se faz necessário entender que fatores determinam esta opção. A sua importância está associada às decisões quanto à definição do perfil do público consumidor desses produtos para que seja formulada a estratégia de marketing adequada. O modelo proposto está dividido em duas fases: quantitativa e qualitativa. Na primeira fase definiram-se as variáveis, os dados foram coletados e as análises de regressão e casualidade realizadas, a fim de encontrar o perfil do consumidor de alimentos orgânicos. Uma vez identificado o perfil, segue-se com a segunda fase, a análise do marketing para o perfil definido e quais as ações devem ser realizadas para melhor atender a esse público consumidor. Neste contexto, consideraram-se as variáveis renda e nível de formação educacional do consumidor como determinantes na intenção de compra/consumo de alimentos orgânicos, e à área plantada de alimentos orgânicos, um proxy representando o consumo de alimentos orgânicos. Assim, buscou-se identificar a existência de correlação entre o consumo e as variáveis explicativas. Obteve-se como resultado a correlação (R) 0,813 , que representa um alto grau de relação entre as variáveis e o coeficiente de determinação $\left(R^{2}\right) 0,661$, representando o poder explicativo do modelo da regressão. Além disso, aplicou-se o modelo de causalidade em que se verificou a casualidade bilateral entre as variáveis. Dessa forma, o modelo responde à definição do perfil do consumidor de produtos orgânicos. Após definir qual o perfil do consumidor de alimentos orgânicos, tendo como base as variáveis limitadas pelo estudo, o próximo passo determinado pelo modelo é a formulação da estratégia de Marketing destinada ao público alvo identificado.

Palavras-chave: Consumo de produtos orgânicos; Correlação; Perfil do consumidor.

\section{Model of consumer profile analysis of organic products to define marketing strategy}

The objective of this study is to propose a model of analysis that identifies the profile of the consumer of organic foods and with the result found traces the marketing strategy directed to this consuming public. Since in the current perspective of the consumer in choosing healthy foods it is necessary to understand what factors determine this option. Its importance is associated to the decisions regarding the definition of the consumer profile of these products in order to formulate the appropriate marketing strategy. The proposed model is divided into two phases: quantitative - qualitative. In the first phase the variables were defined, data were collected and regression and chance analysis were performed to find the consumer profile of organic foods. Once the profile is identified, it follows with the second phase, the marketing analysis for the defined profile and what actions should be taken to better serve this consumer audience. In this context, the variables, income and education level of the consumer were considered, as determinants in the intention to buy / consume organic foods. And to the planted area of organic food, a proxy representing the consumption of organic food. Thus, we sought to identify the existence of a correlation between consumption and explanatory variables. The correlation (R) 0.813 was obtained, which represents a high degree of relationship between the variables and the coefficient of determination $\left(R^{2}\right) 0.661$, representing the explanatory power of the regression model. In addition, the causality model was applied in which the bilateral causality between the variables was verified. In this way, the model responds to the definition of the consumer profile of organic products. After determining the consumer's profile of organic foods based on the variables limited by the study, the next step determined by the model is the formulation of the Marketing strategy aimed at the identified target public.

Keywords: Consumption of organic products; Correlation; Consumer profile.

Topic: Marketing e Estratégias Mercadológicas

Reviewed anonymously in the process of blind peer.

Paulo Eduardo Santos Ferreira

Universidade Federal Rural de Pernambuco, Brasil

http://lattes.cnpq.br/4448069057035247

pauloesferreira@yahoo.com.br

André Marques Cavalcanti Filho

Universidade Federal Rural de Pernambuco, Brasil

http://lattes.cnpq.br/5337128521260089

andremarques2008@gmail.com
Received: 04/05/2017

Approved: 03/07/2017
Referencing this:

FERREIRA, P. E. S.; FILHO, A. M. C.. Modelo de análise do perfil do consumidor de produtos orgânicos para definição da estratégia de marketing. Revista Brasileira de Administração Científica, v.8, n.2 p.42-52, 2017. DOI: http://doi.org/10.6008/SPC2179$\underline{684 X .2017 .002 .0003}$ 


\section{INTRODUÇÃO}

A procura e o interesse pela alimentação saudável vêm crescendo a números consideráveis. Tal interesse está relacionado com a importância por saber de onde vêm os alimentos e quais os impactos à saúde e ao meio ambiente (CESCHIM et al., 2009) com duplo apelo, modo de vida saudável e respeito à natureza, esses alimentos passaram a conquistar as prateleiras dos supermercados, a produção das grandes empresas e cada vez mais consumidores.

Dentre os alimentos saudáveis estão os alimentos orgânicos, os quais em sua produção não são permitidas substâncias que agridam a saúde humana e o meio ambiente, havendo o uso responsável do solo, da água, do ar e dos demais recursos naturais. Segundo as estatísticas do censo agropecuário de 2006, do Instituto Brasileiro de Geografia e Estatísticas (IBGE, 2009), 60\% da produção brasileira são destinados para exportação, principalmente para os EUA, União Europeia e Japão. As vendas de alimentos orgânicos nos Estados Unidos aumentaram de aproximadamente US\$11bi em 2004, para cerca de US \$ 27 bilhões em 2012 (DIAS et al., 2015).

Os EUA têm como principal canal de venda o supermercado, valorizando a rastreabilidade e a certificação dos alimentos (CUNHA, 2010). No mercado brasileiro há uma tendência de crescimento nas vendas nos supermercados (JUNIOR et al., 2014), mas há uma participação das feiras de alimentos orgânicos. Entretanto, segundo pesquisa do Instituto Brasileiro de Defesa do Consumidor (IDEC, 2010), a diferença de preços entre pontos de venda pode chegara $463 \%$.

Assim, pesquisas no Brasil e no mundo buscam identificar quais fatores motivam o consumo de produtos orgânicos. Busca-se então determinar o perfil do consumidor diante das ofertas desses produtos no mercado atual. Uma vez encontrado o perfil do consumidor, procura-se definir a estratégia de marketing adequada para atingir o consumidor de produtos orgânicos. Neste artigo, busca-se, através de um modelo, que será dividido em duas fases: quantitativa, que identifica a existência de correlação entre as variáveis: área plantada de alimentos orgânicos, que para este estudo representará o consumo de produtos orgânicos, o número de concluintes no ensino superior e a renda bruta nacional; e a qualitativa, com elaboração da estratégia de marketing direcionada para o perfil consumidor encontrado.

\section{REVISÃO TEÓRICA}

\section{Alimentos Orgânicos}

A produção de orgânicos difere da convencional não apenas pela ausência de agrotóxico, transgênicos, fertilizantes sintéticos e aditivos, mas por todo um conjunto de cuidados com o meio ambiente. Sua regulamentação, no entanto, deu-se com a publicação do Decreto n.6323 de 2007 . As atividades pertinentes ao desenvolvimento da agricultura orgânica, definidas pela Lei no 10.831, de 23 de dezembro de 2003, ficam disciplinadas por este Decreto, sem prejuízo do cumprimento das demais normas que estabeleçam outras medidas relativas à qualidade dos produtos e processos. A cultura e comercialização dos produtos orgânicos no Brasil foram aprovadas pela Lei n.10831 de 2003. 
Considera-se sistema orgânico de produção agropecuária todo aquele em que se adotam técnicas específicas, mediante a otimização do uso dos recursos naturais e socioeconômicos disponíveis e o respeito à integridade cultural das comunidades rurais, tendo por objetivo a sustentabilidade econômica e ecológica, a maximização dos benefícios sociais, a minimização da dependência de energia não-renovável, empregando, sempre que possível, métodos culturais, biológicos e mecânicos, em contraposição ao uso de materiais sintéticos, a eliminação do uso de organismos geneticamente modificados e radiações ionizantes, em qualquer fase do processo de produção, processamento, armazenamento, distribuição e comercialização, e a proteção do meio ambiente.

O cuidado com o manejo, o uso responsável dos recursos naturais e o respeito aos aspectos ambientais, sociais, econômicos e culturais estão fazendo o Brasil se destacar na produção de alimentos orgânicos, uma vez que há uma preocupação cada vez maior com a segurança dos alimentos e o cuidado com o meio ambiente, ou seja, com a saúde e o bem-estar.

\section{Pesquisas sobre comportamento do consumidor de alimentos orgânicos}

O IDEC (2015) apresenta o resultado da pesquisa referente ao conceito de produto orgânico, sua frequência de compra e sua relação com variáveis de vulnerabilidade social, principalmente de renda e de escolaridade. Os consumidores foram consultados em duas regiões da capital paulista - uma área nobre e de baixa vulnerabilidade social (nos bairros Jardim Paulista e Higienópolis) e outra mais periférica, de menor renda e maior vulnerabilidade social (Itaquera), e concluíram que a escolha por consumir o alimento orgânico vai além do preço, e que vai mais pelo grau de escolaridade e pelo entendimento do conceito de orgânico.

Sampaio (2012) pesquisou dois grupos focais e um survey com 560 pessoas na região de Belo Horizonte, comprovando que não apenas as crenças, o meio ambiente e os hábitos de alimentação saudáveis que influenciam positivamente na intenção de compra/consumo de alimentos orgânicos, mas também, os atributos percepção do preço pago pelo alimento orgânico, percepção da marca de origem orgânica, percepção da qualidade e a disponibilidade do alimento orgânico devem ser consideradas e exercem influências na compra/consumo dos alimentos saudáveis.

Barrena et al. (2010) pesquisou na região da Navarra, na Espanha, dois grupos de consumidores de alimentos orgânicos, os de consumo regular e os ocasionais, dos quais $77,4 \%$ dos consumidores regulares e $86,8 \%$ dos ocasionais têm nível superior de escolaridade. Yin et al. (2010) pesquisou 432 consumidores chineses de 3 cidades e concluíram que a renda, o preço do alimento e o entendimento do conceito do alimento interfere diretamente na decisão de compra.

Aertsens et al. (2011), numa pesquisa em uma feira na Bélgica, verificou que os consumidores de alimentos orgânicos valorizam o meio ambiente, e percebem estes como mais saudáveis. Entretanto, o preço e falta de disponibilidade em determinadas épocas, uma vez que os alimentos orgânicos respeitam as safras, são barreiras para o consumo. Hsieh et al. (2011) concluiu que nos Estados Unidos os consumidores de alimentos orgânicos não são vulneráveis as mudanças de preço, têm uma maior preocupação com a qualidade dos alimentos e compram em supermercados. 


\section{Teste de Casualidade de Granger}

Granger (1969) assume que o futuro não pode causar o passado nem o presente. Por exemplo, se o evento $A$ ocorre depois do evento $B$, sabe-se que $A$ não pode causar $B$. Ao mesmo tempo, se $A$ ocorre antes que $B$, isso não significa que $A$ necessariamente cause B. O exemplo clássico são as previsões de chuva do meteorologista. $\mathrm{O}$ fato de a previsão ocorrer primeiro do que a chuva não implica que o meteorologista cause a chuva. Na prática, o que se tem são duas séries temporais A e B e tendo o interesse em saber se A precede $B$, ou B precede A, ou se A e B ocorrem simultaneamente.

Essa é a essência do teste de causalidade de Granger, que não se propõe a identificar uma relação de causalidade no seu sentido de endogeneidade (MADDALA, 1992). O teste de causalidade de Granger assume que a informação relevante para a predição das respectivas variáveis $\mathrm{X}$ e $\mathrm{Y}$ está contida apenas nas séries de tempo sobre essas duas variáveis. Dessa forma, uma série de tempo estacionária X causa, no sentido de Granger, uma outra série estacionária Y se melhores predições estatisticamente significantes de Y podem ser obtidas ao incluir valores defasados de $\mathrm{X}$ aos valores defasados de $\mathrm{Y}$. Em termos mais formais, o teste envolve estimar as seguintes regressões: $X_{t}=\sum a_{i} y_{t-i}+\sum b_{i} x_{t-i}+u_{1 t}$ e $Y_{t}=\sum c_{i} x_{t-i}+\sum d_{i} y_{t-i}+u_{2 t}$, onde $u_{i t}$ são os resíduos não correlacionados.

A primeira equação postula que valores correntes de $\mathrm{X}$ estão relacionados a valores passados do próprio $\mathrm{X}$, assim como a valores defasados de $\mathrm{Y}$. Na segunda, por outro lado, postula um comportamento similar para a variável Y. Após a estimação, pode-se distinguir quatro casos diferentes tipos de causalidade: unilateral de $Y$ para $X$ : quando os coeficientes estimados em (1) para a variável defasada $Y$ é conjuntamente diferente de zero $\sum a_{i} \neq 0$, e quando o conjunto de coeficientes estimados em (2) para a variável X não forem estatisticamente diferentes de zero $\sum d_{i}=0$.

Continuadamente, unilateral de $\mathrm{X}$ para $\mathrm{Y}$ : quando o conjunto de coeficientes defasados para a variável Y na equação (1) não for estatisticamente diferente de zero $\sum a_{i}=0$ e o conjunto de coeficientes defasados para a variável $\mathrm{X}$ em (2) o for $\sum d_{i} \neq 0$; bicausalidade ou simultaneidade: quando os conjuntos de coeficientes defasados de $\mathrm{X}$ e $\mathrm{Y}$ forem estatisticamente diferentes de zero em ambas as regressões; independência: quando, em ambas as regressões, os conjuntos de coeficientes defasados de $X$ e $Y$ não forem estatisticamente diferentes de zero.

\section{Marketing}

Para Kotler et al. (2006), o marketing envolve a identificação e a satisfação das necessidades humanas e sociais. Ou seja, para Neves et al. (2007) trata-se de um processo que visa satisfazer as necessidades das partes através da troca, suprindo e auferindo lucro com as transações. Corroborando com os autores supracitados, Czinkota (2001) conceitua marketing como o processo de planejamento e execução de concepção, fixação de preços, promoção e distribuição de ideias, bens e serviços para criar trocas que satisfaçam as metas individuais e globais.

A alimentação saudável e consciente, sustentabilidade e respeito ao planeta vêm exigindo das empresas mudanças e adequações para atender a esse exigente mercado consumidor. Os consumidores que 
manifestam preocupação com o meio ambiente e adotam comportamento coerente com as questões sociais e ambientais são qualificados como "consumidores verdes ou ecológicos ou consumidores conscientes ecologicamente" (CARVALHAIS, 2008).

Segundo Prado et al. (2011) nesse processo o marketing ganha importância, como uma forma das empresas estimularem e influenciarem os consumidores a comprarem produtos que sejam ecologicamente corretos. Assim, cabe à empresa fornecer respostas convincentes, reconhecendo a crescente sensibilidade do mercado às temáticas como a sustentabilidade, empenhando-se a atingir resultados positivos a favor do ambiente.

\section{METODOLOGIA}

O presente estudo é uma pesquisa de métodos mistos, uma abordagem da investigação que combina ou associa as formas qualitativa e quantitativa (CRESWELL, 2010). Creswell (2010) aponta as quatro decisões que fazem parte da seleção de uma estratégia de investigação com métodos mistos: Qual é a sequência de coleta de dados quantitativos e qualitativos?; Que prioridade será dada à coleta e à análise de dados quantitativos e qualitativos?; Em que estágio serão integrados os dados e os resultados quantitativos e qualitativos?; e Será utilizada uma perspectiva teórica global?.

Com base neste questionamento, o estudo seguirá a sequência quantitativa - qualitativa. Na primeira fase serão definidas as variáveis, os dados serão coletados e serão realizadas as análises dos dados, de regressão e de casualidade, a fim de encontrar o perfil do consumidor de alimentos orgânicos. Uma vez identificado, segue com a segunda fase, a análise do marketing para o perfil definido e quais as ações deverão ser realizadas para melhor atender a esse público consumidor. A prioridade será dada a análise dos dados qualitativos, porque dela depende a identificação do perfil do consumidor de alimentos orgânicos. E, por conseguinte, o Marketing para este público.

Os resultados quantitativos e qualitativos serão integrados na transição da primeira fase para segunda fase da pesquisa, quando as informações encontradas na fase 1 serão usadas para as ações da fase 2. Como perspectiva teórica será desenvolvido um modelo de análise. A seguir, são apresentadas as características do modelo proposto.

\section{Modelo proposto}

Segundo Sayão (2001), um modelo é uma criação cultural, um 'mentefato', destinado a representar uma realidade, ou alguns de seus aspectos, a fim de torná-los descritíveis qualitativa e quantitativamente e, algumas vezes, observáveis. Para Justi (2006), um modelo é uma cópia da realidade que é constituída através de objetos, acontecimentos, processos ou sistemas, criado para um fim específico.

Assim, com base nos autores, é possível destacar como propriedade comum ao modelo a capacidade de representar. Tal representação se trata de uma estrutura a ser seguida que visa explicar algo. Na figura 1, temos a ilustração do fluxo representativo do modelo proposto que tem início com o perfil do público 
consumidor de alimentos orgânicos e finaliza com a análise do marketing adequado para este público consumidor.

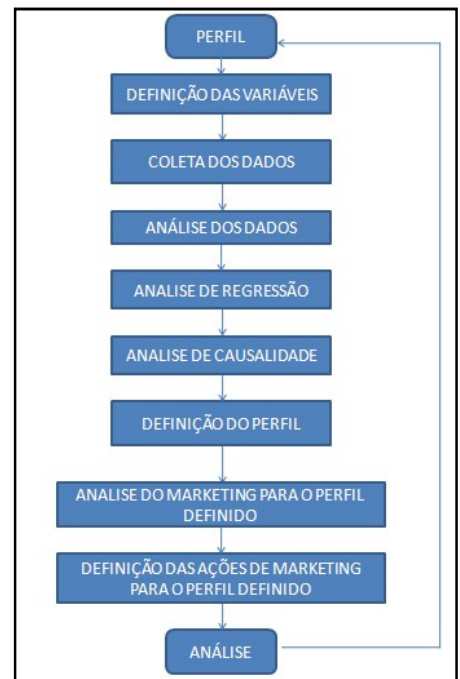

Figura 1: Fluxo representativo do modelo proposto.

\section{Definição das Variáveis e Coleta dos Dados}

Como fonte de informação foi selecionada três séries no tempo, dos anos 2000 até 2013, conforme tabela 1. A variável área plantada de alimentos orgânicos no Brasil, unidade de medida em hectare, obtidas nos anuários para este estudo foi considerada como uma proxy, representando o consumo de alimentos orgânicos. Para a variável formação, o número de concluintes no ensino superior no Brasil, obtidos nos relatórios técnicos INEP (2015). E para renda do Brasil, foi realizado um recorte na série da Renda Nacional Bruta (IPEADATA, 2015), a fim de adequação no tempo com as outras variáveis do estudo.

\section{Análise: dados, regressão e causalidade}

O modelo proposto baseia-se em análise de correlação a partir de regressão linear, a fim de conhecer a correlação entre as variáveis, obtendo-se um modelo que permita fazer previsões de consumo na medida em que se tem conhecimento do comportamento das variáveis renda e nível de formação acadêmica. Como recurso para análise foi utilizado o software PHstat da Universidade Prince Hall, versão 6.0.Para investigar a causalidade entre as variáveis foi usado o modelo de Causalidade de Granger e, como recurso para análise o software econométrico RATS, versão 7.0. Como o teste proposto por Granger visa superar as limitações da correlação entre as variáveis, e o SPSS não contém a opção deste, justifica-se o uso dos dois softwares.

\section{Área}

Na tabela 2 é apresentado o resultado da análise da regressão, tomando a variável dependente o nível de consumo de orgânicos no Brasil, usando como variáveis explicativas o número de formandos em cursos de nível superior e renda do brasileiro. Os dados utilizados estão apresentados na tabela 1, na qual foi excluído o ano de 2013 por se tratar de um outlier, justificado por ser um dado ainda não consolidado. 
O modelo obtido então pode ser representado pela equação 3: $Y_{t}=442557,0989+0,436 x_{1 t}+$ $0,4554 x_{2 t}$, onde: $Y$ é a variável hectares de área plantada naquele ano (variável dependente); $X_{1}$ é a variável quantidade de formandos em curso de nível superior naquele ano - (variável explicativa); e $X_{2}$ é a variável renda dos habitantes - (variável explicativa). A partir da Tabela 1 utilizou-se o IBM SPSS na busca da determinação da correlação entre área plantada de orgânico com formação e renda. Com os resultados da análise de correlação apresentado na Tabela 2.

Tabela 1: Séries no Tempo.

\begin{tabular}{|l|l|l|l|}
\hline Ano & Área Plantada Hectares & Formação Hab. & Renda R\$ \\
\hline 2000 & 100.000 & 352.305 & $1.146 .893,0000$ \\
\hline 2001 & 100.000 & 395.988 & $1.256 .632,0000$ \\
\hline 2002 & 803.180 & 466.260 & $1.425 .886,0000$ \\
\hline 2003 & 275.576 & 528.223 & $1.644 .806,0000$ \\
\hline 2004 & 841.769 & 626.617 & $1.883 .017,0000$ \\
\hline 2005 & 803.180 & 717.858 & $2.085 .653,0000$ \\
\hline 2006 & 887.637 & 736.829 & $2.310 .898,0000$ \\
\hline 2007 & 842.000 & 756.799 & $2.606 .533,0000$ \\
\hline 2008 & 880.000 & 800.318 & $2.960 .428,0000$ \\
\hline 2009 & 1.765 .793 & 826.928 & $3.175 .328,0000$ \\
\hline 2010 & 1.765 .793 & 829.286 & $3.701 .921,8139$ \\
\hline 2011 & 1.765 .793 & 865.161 & $4.064 .885,2541$ \\
\hline 2012 & 1.765 .793 & 876.091 & $4.333 .720,0941$ \\
\hline 2013 & 687.040 & 829.938 & $4.760 .090,0034$ \\
\hline
\end{tabular}

Como se pode observar na tabela 2, que traz uma 'análise de regressão', que o $R^{2}$ é de 0,81 , indicando que o modelo de previsão é consistente, cabendo verificar se há alguma relação de causalidade que possa dar uma maior consistência a representação do fenômeno estudado pelas variáveis propostas, conforme afirmando por Granger (1969).

Tabela 2: Analise de Regressão.

\begin{tabular}{|c|c|c|c|c|}
\hline \multicolumn{2}{|l|}{ RegressionStatistics } & & & \\
\hline Multiple R & 0,9050 & & & \\
\hline R Square & 0,8191 & & & \\
\hline Adjusted R Square & 0,7789 & & & \\
\hline Standard Error & 276899,7539 & & & \\
\hline \multirow[t]{2}{*}{ Observations } & 12 & & & \\
\hline & Coefficients & Standard Error & t Stat & P-value \\
\hline Intercept & $-442557,0989$ & 469949,9081 & $-0,9417$ & 0,3709 \\
\hline a1 & 0,4136 & 1,2532 & 0,3300 & 0,7489 \\
\hline $\mathrm{a} 2$ & 0,4554 & 0,1959 & 2,3242 & 0,0452 \\
\hline
\end{tabular}

Nesta etapa verifica-se se há causalidade entre as variáveis, buscando-se uma relação em que a variável $X$ causa a variável $Y$, então mudanças em $X$ devem preceder temporalmente mudanças em $Y$. Assim, testam-se as 4 condições estabelecidas por Granger (1969) de causalidade unilateral de X para Y, de Y para $X$, bilateral e independente através da aplicação das regressões, utilizando-se das equações (1) e (2) conforme apresentado nas tabelas 3 e 4 .

Tabela 3: Análise de Causalidade de X para Y.

\begin{tabular}{|l|l|}
\hline RegressionStatistics \\
\hline Multiple R & 0,9214 \\
\hline R Square & 0,8490 \\
\hline Adjusted R Square & 0,8188 \\
\hline Standard Error & 264479,2123 \\
\hline
\end{tabular}




\begin{tabular}{|l|l|l|l|l|}
\hline Observations & 13 & \multicolumn{1}{l|}{} & P-value \\
\hline Intercept & Coefficients & Standard Error & & $-1,5413$ \\
\hline Formação Hab & $-544297,3999$ & 353147,2336 & 0,1543 \\
\hline renda & 0,6290 & 1,0434 & 0,6028 & 0,5600 \\
\hline
\end{tabular}

Tabela 4: Análise de Causalidade de Y para X.

\begin{tabular}{|c|c|c|c|c|}
\hline \multicolumn{2}{|l|}{ RegressionStatistics } & & & \\
\hline Multiple R & 0,8967 & & & \\
\hline R Square & 0,8040 & & & \\
\hline Adjusted R Square & 0,7201 & & & \\
\hline Standard Error & 282343,0746 & & & \\
\hline \multirow[t]{2}{*}{ Observations } & 11 & & & \\
\hline & Coefficients & Standard Error & t Stat & P-valu \\
\hline Intercept & $-120402,9329$ & 503503,3263 & $-0,2391$ & 0,8179 \\
\hline Intercept & $-0,2647$ & 0,3472 & $-0,7624$ & 0,4707 \\
\hline Formação Hab & $-0,3733$ & 1,3872 & $-0,2691$ & 0,7956 \\
\hline renda & 0,7147 & 0,3026 & 2,3618 & 0,0502 \\
\hline
\end{tabular}

Conforme verificado com a aplicação das regressões utilizando as equações 1 e 2 com a aplicação dos dados, usando o aplicativo PHstat, obtêm-se as saídas dadas pelas tabelas 3 e 4 . Realizando a sua análise a partir do $R^{2}$ de 0,81 da tabela 3 e $R^{2}$ de 0,72 ,aceita-se a condição da indicação de bilateralidade da causalidade para as variáveis analisadas.

\section{Definição do perfil}

A aplicação do método de análise de regressão multivariada para estabelecimento de correlação entre consumo de produtos orgânicos, renda e nível de formação educacional, determinou uma significativa relação entre as variáveis, indicando que a intenção de compra/consumo de alimentos orgânicos está relacionada com a renda e a formação educacional do consumidor.

\section{Análise do Marketing para o perfil definido}

Conforme relatado anteriormente, o modelo é dividido em duas fases, quantitativa e qualitativa. $\mathrm{Na}$ fase 1, através de métodos quantitativos, foi definido o perfil do consumidor de alimentos orgânicos. A partir daí inicia a fase de transição do modelo, da 1 para 2, da quantitativa para qualitativa, com vistas de analisar e definir o marketing ou as estratégias de marketing adequadas para o público consumidor de alimentos orgânicos. Entretanto, antes se faz necessário conhecer quem é o consumidor de produtos orgânicos. Segundo Calzada (2008), o consumidor orgânico se divide em três:

Consumidor 'ecológico' e militante: conscientizado e informado, esse cliente sabe bem o que quer e faz suas compras com atenção e cuidado: olha o rótulo do produto, verifica se tem algum selo de certificação, quer saber a origem do produto, como foi produzido e quer garantias. É o tipo de consumidor que questiona a ausência na indústria de embalagens degradáveis para os produtos orgânicos. Procura fugir da alimentação industrializada e de produtos que tenham resíduos químicos;

Consumidor que quer se iniciar em um novo estilo de vida e de consumo: procura se informar e sabe que o padrão de sua alimentação pode melhorar em qualidade. Compra o produto orgânico, pela recomendação de outras pessoas que já o conhecem, pois está acostumada a consumir apenas as marcas tradicionais do mercado. Nessa mesma categoria, estão aqueles que buscam um novo estilo de consumo por razões de doença ou intoxicação. 
Procuram melhorar a qualidade de sua alimentação, orientados por médicos e nutricionistas;

Consumidor gourmet: procura o produto de alta qualidade, orgânico ou não. Os produtos orgânicos o interessam na medida em que lhe dão a garantia de um produto fresco e saboroso. Para esse consumidor, o produto processado não pode ter conservantes ou aditivos de qualquer espécie. Orienta-se pelo sabor e bom gosto, pois está acostumado a degustar e escolher o melhor. É tanto ou mais exigente que o consumidor militante, ainda que por motivos diferentes.

O modelo proposto vai considerar como consumidor de alimentos orgânicos apenas o consumidor 'ecológico' e militante. Consumidor esse que dá significado ao consumir. Tem esse consumo como um estilo de vida, e um nível de entendimento que reflete nas escolhas quanto à forma de gastar seu tempo e seu dinheiro (SALOMON, 2011), conforme representam as relações contidas na figura 2: pessoas, produtos e ambientes combinados para expressar um estilo de consumo.

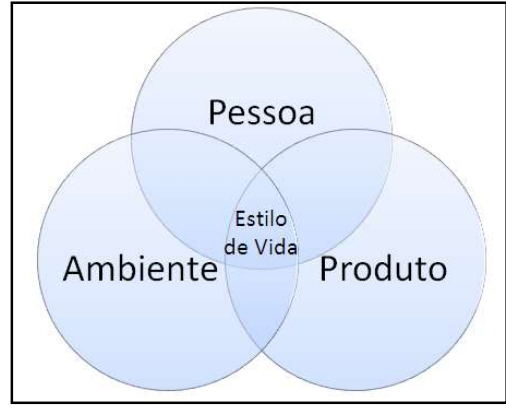

Figura 2: Relação Pessoa, Produto e Ambiente. Fonte: Salomon (2011).

E para atender a esse público consumidor de alimentos orgânicos uma estratégia adequada é a de Marketing de Nicho, que, segundo Kotler (2009), os nichos descrevem conjuntos menores de clientes que apresentam necessidades mais definidas ou combinações únicas de necessidades. Cientes e consciente do valor deste tipo de alimento, os consumidores têm disponibilidade em pagar preços Premium para os orgânicos (NEVES,2007).

\section{Definição das ações de marketing para o perfil definido}

Brasil (2015) identificou que no conjunto das 27 cidades brasileiras pesquisadas, a frequência de adultos obesos foi de $16,8 \%$. Entre os homens, a frequência da obesidade tendeu a aumentar com a idade até os 44 anos e, entre as mulheres, até os 54 anos. A frequência de obesidade tendeu a diminuir com o aumento do nível de escolaridade em ambos os sexos.

Oliveira (2014) corrobora quando identificou que as características das famílias afetam a probabilidade de realizar despesas com alimentos orgânicos ou light/diet e a maior probabilidade de consumo está no estrato mais elevado de $\mathrm{R} \$ 3.500$ a $\mathrm{R} \$ 5.600$ mensais per capita. Assim, as ações de marketing devem estar direcionadas para o consumidor que possui níveis de escolaridade que sugerem ao indivíduo um maior nível de conhecimento e de consciência, que amplie a capacidade e a criticidade para escolher os alimentos mais adequados para o seu consumo. 
Assim um cenário favorável vai ao encontro desse nicho de pessoas que estão aderindo a uma alimentação e estilo de vida voltada ao bem estar, a ética alimentar e ao cuidado com o meio ambiente. Acessos à informação e à cultura levaram a uma mudança qualitativa no consumo de alimentos, resultando em impacto similar a melhora nos níveis de escolaridade. Cientes e consciente do valor deste tipo de alimento, os consumidores têm disponibilidade em pagar preços Premium para os orgânicos (NEVES, 2007). As ações de marketing para o perfil definido deve trabalhar a imagem do sistema orgânico no mercado focando no conceito de saúde humana, social e ambiental, que, segundo Azevedo (2011),a melhor estratégia de marketing para um produto alimentar atualmente é ter um parecer sobre saúde.

\section{CONCLUSÕES}

Com base nos resultados apresentados, nos quais os fatores, renda e formação determinam significativamente a intenção de compra/consumo de alimentos orgânicos e quais as relações desses fatores entre si foi aplicado o método de análise de regressão multivariada para estabelecer uma correlação entre consumo de produtos orgânicos, renda e nível de formação educacional. Para se confirmar a existência da interdependência entre as variáveis estudadas foi realizada, em conjunto, a análise da causalidade, em que foi identificada a bilateralidade na interdependência entre as variáveis. A importância dessa constatação, a partir do modelo apresentado, permite estabelecer ações de marketing direcionadas para o público definido pelo modelo, aumentando, assim, a eficácia, e possibilitando estratégias diferenciadas para o público alvo estabelecido pelo modelo.

Desta forma, se a decisão for de elevar o nível de consumo de produtos orgânicos deverão ser dirigidas ações de marketing para o público que possui o perfil aqui definido. Neste sentido, as campanhas devem ser dirigidas à população com nível superior, que possua nível de renda para adquirir o alimento orgânico.

Logo, o consumo de alimento orgânico, representado pelo aumento da área plantada ao longo do tempo, depende da formação educacional e da renda, o que se justifica porque envolve o conhecimento, entendimento e compreensão de que o alimento orgânico é saudável, tem um maior nível de segurança alimentar, sendo certificado por isso, e contribui com a sustentabilidade do planeta. $\mathrm{E}$ isto tem um custo, o que faz esses alimentos sejam mais caros que os alimentos convencionais, o que implica que as pessoas que consomem orgânicos têm poder de compra, assumindo que vale a pena o investimento.

\section{REFERÊNCIAS}

AERTSENS, J.; MONDELAERS, K.; VERBEKE, W.; BUYSSE, J.; HUYLENBROECK, G. V.. The influence of subjective and objective knowledge on attitude, motivations, and consumption of organic food. British Food Journal, Bingley, v.113, n.11, p.1353-1378, 2011.

AZEVEDO, E.. Riscos e controvérsias na construção do conceito de Alimento Saudável: o caso da soja. Revista Saúde Pública, v.45, n.4, p.781-788, 2011.
BARRENA, R.; SÁNCHEZ, M.. Frequency of consumption and changing determinants of purchase decision: from attributes to values in the organics food market. Spanish Journal of Agricultural Research, v.2, n.8, p.251-272, 2010.

BRASIL. Decreto n.6323 de 27 de dezembro de 2007. Institui as atividades pertinentes a agricultura orgânica. Brasília: DOU, 2007 
BRASIL. Lei n.10831 de 23 de dezembro de 2003. Dispõe sobre a agricultura orgânica e dá outras providências. Brasília: DOU, 2003.

BRASIL. VIGITEL Brasil 2014: vigilância de fatores de risco e proteção para doenças crônicas por inquérito telefônico. Brasília: MS, 2015.

CALZADA, M.. Quem é o consumidor orgânico. Mar del Plata: IFOAM, 1998.

CARVALHAIS, R. S.. Atitudes e comportamento dos consumidores diante de produtos com apelo ecológico. Dissertação (Mestrado em Administração) - Faculdades Integradas Pedro Leopoldo, Minas Gerais, 2008.

\section{CASTRO, L. T.; NEVES, F. M. Marketing e Estratégia e} Estratégia em Agronegócios e Alimentos. São Paulo: Atlas, 2003.

CESCHIM, G.; MARCHETTI, R. Z.. O comportamento inovador entre consumidores de produtos orgânicos: uma abordagem qualitativa. In: ENCONTRO NACIONAL DA ASSOCIAÇÃO NACIONAL DE PÓS-GRADUAÇÃO E PESQUISA EM ADMINISTRAÇÃO, 33. Anais. São Paulo, 2009.

CRESWELL, J. W.. Projeto de pesquisa: métodos qualitativos, quantitativos e mistos. Porto Alegre: Artmed, 2010.

CUNHA, C. F. D.. Custo de Transação e Mensuração nas Relações Contratuais entre Supermercado e Produtores Agrícolas Convencionais e Orgânicos no Brasil e EUA. Tese (Doutorado em Administração) - Universidade de São Paulo, São Paulo, 2010.

CZINKOTA, M. R.. Marketing: as melhores práticas. Porto Alegre: Bookman, 2001.

DIAS, V. V.; SCHULTZ, G.; SCHUSTER, M. S.; TALAMINI, E.; RÉVILLION, J. P.. O mercado de alimentos orgânicos: um panorama quantitativo e qualitativo das publicações internacionais. Ambiente \& Sociedade, São Paulo, n.1, p.161-182, jan.-mar. 2015.

GRANGER, C. W. J.. Investigating causal relatinos by econometric models and cross-spectral models. In: GRANGER, C. W. J.. Econometrica 34, p.541-551, 1969.

HSIEH, M. F.; STIEGERT, K. W.. Store format choice in organic food consumption. Journal of Agricultural and Applied Economics, v.92, n.2, p.307-313, 2011.

IBGE. Instituto Brasileiro de Geografia e Estatística. Censo agropecuário 2006: Brasil, grandes regiões e unidades da federação. Rio de Janeiro: IBGE, 2009.

IDEC. Instituto Brasileiro de Defesa do Consumidor. Pesquisa Alimentos: muito além do preço. Revista IDEC, n.192, p.1825, 2015.
IDEC. Instituto Brasileiro de Defesa do Consumidor. Revista IDEC, n.142, p.15-20, 2010.

INEP. Instituto Nacional de Pesquisas Educacionais Anísio Teixeira. Censo da educacao superior: resumos tecnicos: 2000-2013. Brasília: INEP, 2015.

IPEADATA. Base de dados mantida pelo Instituto de Pesquisas Econômica Aplicada (IPEA). ISSN Renda Nacional Bruta. Brasília: IPEA, 2000.

JUNIOR, S. S. B.; NETO, A. R. V.; MORAES, R.. Atributos de estilo de vida do consumidor relacionados ao consumo de produtos orgânicos no varejo especializado. Revista Brasileira de Marketing, v.13, n.5, 2014.

JUSTI, R.. La enseñanza de ciencias basada en la elaboración de modelos. Enseñanza de las Ciências, v.24, n.2, p.173-184 2006.

KOTLER, P.. Marketing para o Século XXI: como criar, conquistar e dominar mercados. São Paulo: Ediouro, 2009.

KOTLER, P.; KELLER, L.. Administração de Marketing. 12 ed. São Paulo: Pearson Prentice Hall, 2006.

MADDALA, G. S.. Introduction to Econometrics. 2 ed. Nova York: MacMillan, 1992.

NEVES, F. M.. Agronegócio e desenvolvimento sustentável: uma agenda para liderança mundial na produção de alimentos e bioenergia. São Paulo: Atlas, 2007.

OLIVEIRA, F. C. R.. Alimentos Normais, light/diet e orgânicos: o consumo segundo as classes econômicas e suas elasticidades-renda. Tese (Doutorado em Ciências) Universidade de São Paulo, Piracicaba, 2014.

PRADO, R. A. D. P.; SILVA, M. A.; JUNQUEIRA, M. C.; ALMEIDA. L. N. N.. A influência do marketing verde nos hábitos de consumo dos jovens universitários dos cursos de administração: estudo em instituições de ensino superior (IES). Revista Brasileira de Marketing, São Paulo, v.10, n.2, p.126-145, 2011.

SALOMON, M. R.. O comportamento do consumidor: comprando, possuindo e sendo. 9 ed. Porto Alegre: Bookman, 2011.

SAMPAIO, D. O.. Comportamento do consumidor de alimentos orgânicos:um estudo sobre crenças, atributos e grupos de referência. Tese (Doutorado em Administração) Universidade Federal de Minas Gerais, Belo Horizonte, 2012.

SAYÃO, L. F.. Modelos teóricos em ciências da informação: abstração e métodos científicos. Ciência da Informação, Brasília, v.30, n.1, p.82-91, 2001.

YIN, S.; WU, L.; DU, L.; CHEN, M.. Consumers purchase intention of organic food in China. Journal Sci Food Agricultural, v.90, p.1361-1367, 2010. 